\title{
Motion style acupuncture therapy for shoulder pain: a randomized controlled trial
}

This article was published in the following Dove Press journal: Journal of Pain Research

\author{
Guang-Xia Shi' \\ Bao-Zhen Liu \\ Jun Wang ${ }^{3}$ \\ Qing-Nan Fu' \\ San-Feng Sun ${ }^{2}$ \\ Rui-Li Liang ${ }^{2}$ \\ Jing $\mathrm{Li}^{3}$ \\ Jian-Feng $T^{\prime}$ \\ Cheng $\operatorname{Tan}^{3}$ \\ Cun-Zhi Liu ${ }^{4}$
}

'Acupuncture and Moxibustion Department, Beijing Hospital of Traditional Chinese Medicine Affiliated to Capital Medical University, Beijing 100010 , China; ${ }^{2}$ Acupuncture and Moxibustion Department, Beijing Huairou District Hospital of Traditional Chinese Medicine, Beijing 101400, China; ${ }^{3}$ Department of Acupuncture and Moxibustion, Dongzhimen Hospital, Beijing University of Chinese Medicine, Beijing I000 I0, China; ${ }^{4}$ Department of Acupuncture and Moxibustion, Dongfang Hospital, Beijing University of Chinese Medicine, Beijing I00078, China

Correspondence: Cun-Zhi Liu Department of Acupuncture and Moxibustion, Dongfang Hospital, Beijing University of Chinese Medicine, No. 6 Fangxingyuan Ist Block, Fengtai District, Beijing 100078, China

Tel/fax +86 I0 67678865

Email Icz623780@।26.com
Background: Strategies for preventing the persistence of pain and disability beyond the acute phase in shoulder pain patients are critically needed. Conventional acupuncture therapy (CAT) or motion style acupuncture therapy (MSAT) alone results in relative improvements in painful conditions in shoulder pain patients; combined interventions may have more global effects. The aim of this study is to evaluate the efficacy and safety of MSAT vs CAT for shoulder pain. Methods: A randomized controlled trial using a factorial design was conducted from January 2014 to December 2015. Patients with a primary complaint of one-sided shoulder pain participated at three study sites. Eligible individuals were randomly assigned to receive MSAT plus minimal CAT (mCAT), CAT plus minimal MSAT (mMSAT), MSAT plus CAT, or mMSAT plus mCAT for 6 weeks in a 1:1:1:1 ratio. The primary outcome was change in shoulder pain intensity (measured using visual analog scale). The secondary outcomes included change in function of the shoulder joint (Constant-Murley score) and the health-related quality of life (Short Form-36 Health Survey). Moreover, perceived credibility of acupuncture was measured using the Treatment Credibility Scale. The outcomes were assessed at baseline and at 6,10, and 18 weeks after randomization. Analysis of covariance with the baseline score adjustment had been used to determine the primary end point. The between-group differences of MSAT vs mMSAT and CAT vs mCAT were estimated, respectively, after tests of interaction between the two-dimensional interventions. All main analyses followed the intention-to-treat principle. Results: A total of 164 patients completed the study. MSAT was superior to mMSAT in alleviating pain intensity at 10 weeks $(P=0.024)$, and it was maintained for 18 weeks $(P=0.013)$. Statistically significant differences were found when comparing MSAT with mMSAT for improvement in shoulder function ( 6 weeks, $P=0.01 ; 10$ weeks, $P=0.006$; and 18 weeks, $P=0.01$ ), physical health ( 10 weeks, $P=0.023$ and 18 weeks, $P=0.015$ ), and mental health ( 18 weeks, $P=0.05$ ). No significant differences were found in CAT when compared with mCAT.

Conclusion: After 18 weeks of treatment, pain and joint functions are improved more with MSAT than with minimal motion style acupuncture or conventional acupuncture in patients with shoulder pain.

Keywords: motion style acupuncture, shoulder pain, acupuncture therapy, randomized controlled trial

\section{Introduction}

Shoulder pain is the third most frequently reported type of musculoskeletal pain. ${ }^{1}$ Its monthly prevalence in the general population is reported to be between $18 \%$ and $31 \%$, whereas its lifetime prevalence ranges between $6.7 \%$ and $66.7 \% .^{2}$ Although the natural history of shoulder pain varies and shoulder injuries are generally self-limiting, 
up to half of the persons who present for care, particularly the elderly, might continue to have pain and/or functional disturbances for up to 2 years after presentation, substantially decreasing their quality of life. ${ }^{3}$ Thus, effective treatments for shoulder pain patients are needed to prevent the persistence of pain and disability beyond the acute phase..$^{4-7}$

Acupuncture therapy has been mostly used for a range of painful and other conditions, including musculoskeletal disorders of the shoulder or other regions; however, high-quality evidence for its efficacy is scant. ${ }^{8}$ Conventional acupuncture, inserting needles into specific points in the body, has been used in China for $>2000$ years. Motion style acupuncture therapy (MSAT) is a relatively novel acupuncture method that has been recently used more often to treat pain in China and South Korea. It is similar to traditional acupuncture in that the needles are inserted at specific acupuncture points, but it is unique in that it requires passive or active movement of the patient's body while the acupuncture needles are retained. Shin et $\mathrm{al}^{9}$ conducted a multicenter, randomized, comparative effectiveness trial to evaluate the effects of MSAT on acute low back pain with severe disability. They found that MSAT has positive effects such as immediate pain relief and the functional recovery of acute low back pain patients. ${ }^{9}$ MSAT or conventional acupuncture alone results in relative improvements in painful conditions in shoulder pain patients; combined interventions may have more global effects.

To our knowledge, there are no previous trials that have studied the effect of a treatment modality that combines acupuncture with passive or active movement of the patient's body compared to MSAT for shoulder pain patients. The purpose of this study was to examine whether MSAT is more effective in reducing pain and disability than minimal MSAT (mMSAT) in people with shoulder pain and whether conventional acupuncture therapy (CAT) is more effective in reducing pain and disability than minimal CAT (mCAT) in people with shoulder pain.

\section{Methods}

\section{Study design and setting}

This was a randomized controlled factorial trial. The main comparisons were between MSAT and mMSAT and between CAT and mCAT (Table S1). The Acupuncture Therapy for Shoulder Pain Trial was conducted between January 1, 2014, and December 31, 2015, at the Beijing Hospital of Traditional Chinese Medicine Affiliated to the Capital Medical University, the Beijing Huairou District Hospital of Traditional Chinese Medicine, the Dongzhimen Hospital, Beijing University of Chinese Medicine, and Dongfang Hospital, Beijing University of Chinese Medicine. The study protocol was approved by the ethics committee of the Beijing Hospital of Traditional Chinese Medicine Affiliated to Capital Medical University (ref: 201315). The protocol, including the statistical analysis plan, has been published previously (ISRCTN61861069, http://www.controlled-trials.com). ${ }^{10}$

This study followed the Declaration of Helsinki and Good Clinical Practice guidelines and was reported according to the Consolidated Standards of Reporting Trials (CONSORT) and Standards for Reporting Interventions in Clinical Trials of Acupuncture (STRICTA) guidelines.

\section{Study population}

Participants with shoulder pain were recruited from hospitals and communities via posters and newspaper advertisements. We included patients who were aged between 25 and 65 years, presented with a primary complaint of one-sided shoulder pain for at least 6 weeks and up to 2 years, obtained a pain score of $\geq 50 \mathrm{~mm}$ on a $100 \mathrm{~mm}$ visual analog scale (VAS), and had normal shoulder radiography results. The physical record of the shoulder included an X-ray to detect osteoarthritis of the glenohumeral joint or other bone pathologies, the results of tests for diminished strength or atrophy of the muscle, and the results of range of passive motion tests (abduction, adduction, rotation, and elevation). The Jobe test and drop arm test were chosen as diagnostic tests by a relevant professional who had rich clinical experience in the diagnosis of rotator cuff tear. Patients who have positive result for one of the two tests were excluded. ${ }^{11,12}$

Patients were excluded if they had pain due to tendonitis; the cervical spine; osteoarthritis of the glenohumeral joint or systemic bone and joint disorders (rheumatoid arthritis); a history of shoulder trauma, shoulder surgery, stroke, ipsilateral breast surgery, heart disease, or severe hypertension; endocrine diseases, such as hyperthyroidism; severe infection; or ongoing, current therapy involving analgesics.

\section{Randomization and masking}

Random allocation was performed if a participant was eligible and had signed the informed consent form. Randomization was stratified by center with a block size of 9 and was unknown to the trial center. The randomization list was generated using the SAS Version 9.1.3 statistical package (SAS Institute Inc., Cary, NC, USA), and patients were randomly assigned to treatment groups in a 1:1:1:1 ratio. A researcher who has no other role in the study implemented the allocation schedule using a centralized telephone randomization procedure. Randomization was concealed and recorded on a secure central database. Randomized allocation 
of the next patient was concealed from the administrators and acupuncturist until the point of randomization.

The acupuncturists were not blinded to the treatments they delivered because acupuncture manipulation made this impossible. During the intervention, the acupuncturist and personnel who collected data and assessed the outcome were segregated immediately after the treatment start and were trained not to exchange information with each other. The patients, trial statisticians, outcome assessors, and data entry personnel were blinded to treatment assignment throughout the study.

\section{Procedure}

Three researchers were trained in clinical interviews and selection criteria performed in the initial assessment, consisting of a detailed examination to check for the clinical diagnosis, patient fulfillment of inclusion criteria, collection of baseline data, description of the study to the patients, and request for written informed consent.

All the procedures used in the present study were reviewed and approved by the local institutional review board. The participants were enrolled via advertisements in the community, media, medical/physical therapy clinics, and acupuncture clinics. Written informed consent was obtained from all the patients. ${ }^{13}$ No economic incentives were offered, but the treatment was free of charge as compensation for participation. The consent and source data were verified by independent clinical monitors.

\section{Treatments}

We developed the trial interventions using a consensus process that reviewed Chinese acupuncture textbooks with the help of an expert panel. Acupuncture was administered in outpatient clinics by conventionally trained acupuncturists (ie, they had an experience of $>15$ years and an acupuncture license from the Ministry of Health of the People's Republic of China) with additional extensive acupuncture training (median, 8 hours; interquartile range, 6-12 hours). They were also trained in minimal acupuncture therapies and were instructed to deliver both in the same context and in the same behaviors.

The same number and type of needles (coated, sterile, single-use acupuncture needles $0.25 \mathrm{~mm} \times 40 \mathrm{~mm}$ and $0.25 \mathrm{~mm} \times 75 \mathrm{~mm}$, Hwato; Suzhou Medical Appliance Factory, Suzhou, China) were used in all treatment groups. With the exception of the insertion site, depth, and manual stimulation, other factors, such as size of needle, retention time, frequency of treatment, intensity of the movement, and number of treatments, were identical among the four acupuncture groups. Additionally, the investigators were instructed to provide the same level of care and attention to all groups of patients. The patients visited the treatment setting to receive the intervention twice weekly for 6 weeks. To better approximate daily clinical practice in the treatment of shoulder pain, the participants who attended $\geq 80 \%$ (10 of 12) of the acupuncture appointments were considered to have completed a full course of treatment. To ensure the consistency of acupuncture treatment, a training workshop was conducted for acupuncturists who were involved in the study from three centers. It included an additional extensive acupuncture training (median, 8 hours; interquartile range, 6-12 hours). An interrater reliability coefficient ( $k$ value) of $>0.80$ was achieved after the completion of training workshop. Two supervisors monitored compliance with the protocol throughout the study. The on-site monitoring was done every 2 months. Patients were allowed to treat shoulder pain with concomitant treatments, and the details of concomitant treatments were documented by the patients.

In this study, eligible individuals were randomly assigned to one of the following four groups: MSAT plus mCAT group, CAT plus mMSAT group, MSAT plus CAT group, and mMSAT plus mCAT group in a 1:1:1:1 ratio. The four groups are as follows:

MSAT plus mCAT: the MSAT for 20 minutes, after removing the needles, the mCAT was performed for another 20 minutes.

CAT plus mMSAT: the CAT for 20 minutes, after removing the needles, the mMSAT was performed for another 20 minutes.

MSAT plus CAT: the MSAT for 20 minutes, after removing the needles, the CAT was performed for another 20 minutes.

mMSAT plus mCAT: the mMSAT for 20 minutes, after removing the needles, the mCAT was performed for another 20 minutes.

We combined two of the four therapies as the interventions given to patients in one group. Table $\mathrm{S} 1$ shows the core components of four different therapies.

\section{Motion style acupuncture therapy}

The patient was asked to remain seated and to relax their shoulder. In this position, the acupuncturist inserted disposable needles to a depth of $10-15 \mathrm{~mm}$ at the subject's "Tiaokou" (ST 38, contralateral), followed by stimulation with rotational movements of the needle in an arc of at least 
$180^{\circ}$ to achieve a strong sense of Deqi (gradually increasing stimulation at ST 38 until a sensation radiates throughout the lower limb). The needle was maintained in that position for 20 minutes and was manipulated for 1 minute every 5 minutes (with a total of four manipulations per session). During the periods of manipulation, the subjects were asked to perform active mobilization of the shoulder, in abduction and internal and external rotations.

\section{Conventional acupuncture therapy}

The subjects received acupuncture at three local acupoints on the shoulder, such as "Jianyu” (LI 15), "Jianliao" (TE 14), and "Jianzhen" (SI 9), namely "Jiansanzhen" in combination with "Binao" (LI 14). The needle was inserted to a depth of $10-15 \mathrm{~mm}$, was maintained in that position for 20 minutes and was manipulated for 1 minute every 5 minutes (with a total of four manipulations per session) to obtain Deqi.

\section{Minimal MSAT}

The needle was inserted at a nonacupoint (located lateral to the shank, $3 \mathrm{~cm}$ below gallbladder 34 , and midway between the gallbladder meridian and the bladder meridian) distal from the shoulder. The needle was inserted to a depth of 3-5 mm using a shallow needling technique without Deqi and was maintained in that position for 20 minutes. In this period, the subjects were asked to perform active mobilization of the shoulder, in abduction and internal and external rotations.

\section{mCAT}

The needles were only superficially inserted $(3-5 \mathrm{~mm}$ in depth) for 20 minutes at predefined nonacupoints around the shoulder: 1) in the anterior axillary fold; 2) in the posterior axillary fold; 3 ) in the shoulder, $2 \mathrm{~cm}$ below "Tianzong" (SI $11)$; and 4) inside the upper arm toward the bicep, "Tianfu" (LU 3), inward $1 \mathrm{~cm}$, between the pericardium meridian and lung meridian. No specific manipulation was used in this process, and the Deqi sensation was not sought.

\section{Outcome measures}

The primary trial outcome was change in pain intensity measured by VAS. The patient placed a perpendicular line on the top of a horizontal or vertical VAS at the point that represented their pain intensity; then, the assessors measured the distance in millimeters with a ruler $(0=$ no pain and $100=$ the most excruciating pain that the patient had ever experienced).
The secondary outcomes included change in function of the shoulder joint, which was assessed using the ConstantMurley score (CMS) with higher scores indicating better function (0-100 scores). The CMS consists of the following four domains: pain, activities of daily living, mobility, and power or strength. Health-related quality of life was assessed using a Chinese version of the Short Form-36 Health Survey (SF-36), which consists of the physical health and mental health subscales. Higher scores indicated a better healthrelated quality of life.

The perceived credibility of acupuncture was assessed by patients after a 6-week acupuncture treatment, using the Treatment Credibility Scale. ${ }^{14}$ The participants scored each response on a scale from 1 (not at all useful/confident) to 5 (very useful/very confident) by answering the following question immediately after treatment: "At this point, how successfully do you think this treatment will alleviate the symptoms that brought you to the treatment?" A higher score indicates greater perceived credibility of treatment.

The participants were advised to report any adverse events that they experienced, including discomfort or bruising at the sites of needle insertion, nausea, or presyncope during treatment.

\section{Sample size}

The analysis was performed using the SPSS software program (SPSS 16.0 KO for Windows). According to the previous study, ${ }^{15} 50 \%$ of reduction compared with baseline in the VAS scores is considered to be clinically relevant. A total of $65 \%$ of responders were verum and $24 \%$ of responders were sham acupuncture. To achieve $90 \%$ power at a two-sided 5\% significance level, a total of 136 participants with data at baseline and at 6 weeks were required. To allow a $20 \%$ dropout over the 6 -week postrandomization period, we planned to recruit 164 participants to the trial, ie, 41 participants per group for each comparison.

\section{Statistical analysis}

$P<0.05$ was considered to be statistically significant. Continuous variables were expressed as mean (SD) and compared by Student's $t$-test or one-way analysis of variance where appropriate. Categorical variables were expressed as number (percentage) and compared using the $\chi^{2}$ test.

The primary analysis followed the intention-to-treat principle. Missing data on VAS score were handled by the last observation carried forward method as prespecified. Analysis of covariance with the baseline score adjustment was used for 
primary end point. The between-group differences of MSAT vs mMSAT and CAT vs mCAT and their corresponding 95\% confidence interval were estimated, respectively, after the test of interaction between the two-dimensional interventions. Sensitivity analysis of pain intensity VAS score at 18 weeks was conducted by a mixed effect model with repeated measurements. Furthermore, per protocol analysis was done to test the reliability of findings on primary outcome.

\section{Results}

A total of 397 participants were considered for enrollment, 285 participants were assessed for eligibility, and 164 participants were randomly assigned. Trial eligibility, recruitment, and follow-up are described in Figure 1. Overall, the follow-up rates (including minimum data collection) were $89.6 \%$ (147) for 6 weeks, $89.6 \%$ (147) for 10 weeks, and 89.6\% (147) for 18 weeks. Table 1 shows the baseline characteristics of the participants. The mean (SD) age of the participants was 53.9 (7.7) years, and $63.4 \%$ were female; the mean (SD) pain and function scores at trial entry were 71.2 (1.3) for pain and 49.7 (11.0) for function. Overall, the differences in participant characteristics across the treatment arms at baseline were small; however, some between-group differences were observed for sex and in the SF-36 physical health subscale.

\section{Main trial results}

The interaction terms for the primary outcome and all other outcomes (Table 2 ) were not statistically significant $(P>0.05$ ); therefore, the treatment effects were evaluated from the main effects model (ie, MSAT vs mMSAT and CAT vs mCAT).

The prespecified primary end point of this study was VAS score of shoulder pain intensity at the end of follow-up (18 weeks). After the adjustment of baseline score, the difference in pain intensity between MSAT and mMSAT groups was -8.28 (95\% CI: $-15.43,-1.13)$.

Table 3 shows treatment effectiveness for outcome measures by main treatment effects. MSAT was superior to mMSAT in alleviating pain intensity at 10 weeks $(P=0.024)$, and this was maintained at 18 weeks $(P=0.013)$. Statistically significant differences were found when comparing MSAT with mMSAT for improvement in shoulder function (6 weeks, $P=0.01$; 10 weeks, $P=0.006$; and 18 weeks, $P=0.01$ ), physical health ( 10 weeks, $P=0.023$ and 18 weeks, $P=0.015$ ), and mental health ( 18 weeks, $P=0.05$ ). Among these differences, only the MSAT comparison reached statistical significance. No statistically significant differences were observed at 6 , 10, and 18 weeks for CAT comparison with mCAT.

\section{Treatment credibility}

The participants rated the credibility of the treatment very highly and almost identically after 12 treatment sessions (Table 4).

\section{Sensitivity analyses}

Although no statistical significance was observed at 10 weeks $(P=0.07)$ on per protocol data, significantly greater reduction in pain intensity was present at 18 weeks $(P=0.045)$ (Table $5)$. The results support the main conclusions of this study.

\section{Adverse events}

Adverse events were reported at 6 weeks. There were six subjects who reported a small hematoma or bleeding, four subjects who experienced discomfort at the sites of needle insertion, and two subjects who had needling pain. A total of 12 side effects were reported by three (7\%) subjects in the MSAT plus CAT group (small hematoma or bleeding), three (7\%) subjects in the CAT group (small hematoma and discomforts), three (7\%) subjects in the MSAT group (small hematoma, bleeding, and needling pain), and three (7\%) subjects in the mMSAT plus mCAT group (small hematoma and discomforts).

\section{Concomitant treatments}

At baseline, 20 (12\%) participants had received other treatment methods for their current episode of shoulder pain (Table 1). During the follow-up period, there was no significant difference in concomitant treatments among the four groups. In the MSAT plus mCAT group, six patients received an oral drug treatment (NSAIDs); in the CAT plus mMSAT group, five patients received an oral drug treatment (NSAIDs), one patient received superficial thermotherapy, and one patient received injections in the shoulder joint; in the MSAT plus CAT group, five patients received an oral drug treatment (NSAIDs) and two patients received superficial thermotherapy; and in the mMSAT plus mCAT group, four patients received an oral drug treatment (NSAIDs) and one patient received physical therapy (massage).

\section{Discussion}

In this multicenter, randomized controlled factorial trial, we found that there is no interaction between MSAT and CAT for all the outcomes. For further analysis, we evaluated whether MSAT was more effective in reducing pain and disability than mMSAT among people with shoulder pain. Our results showed that subjects who received MSAT had significantly 


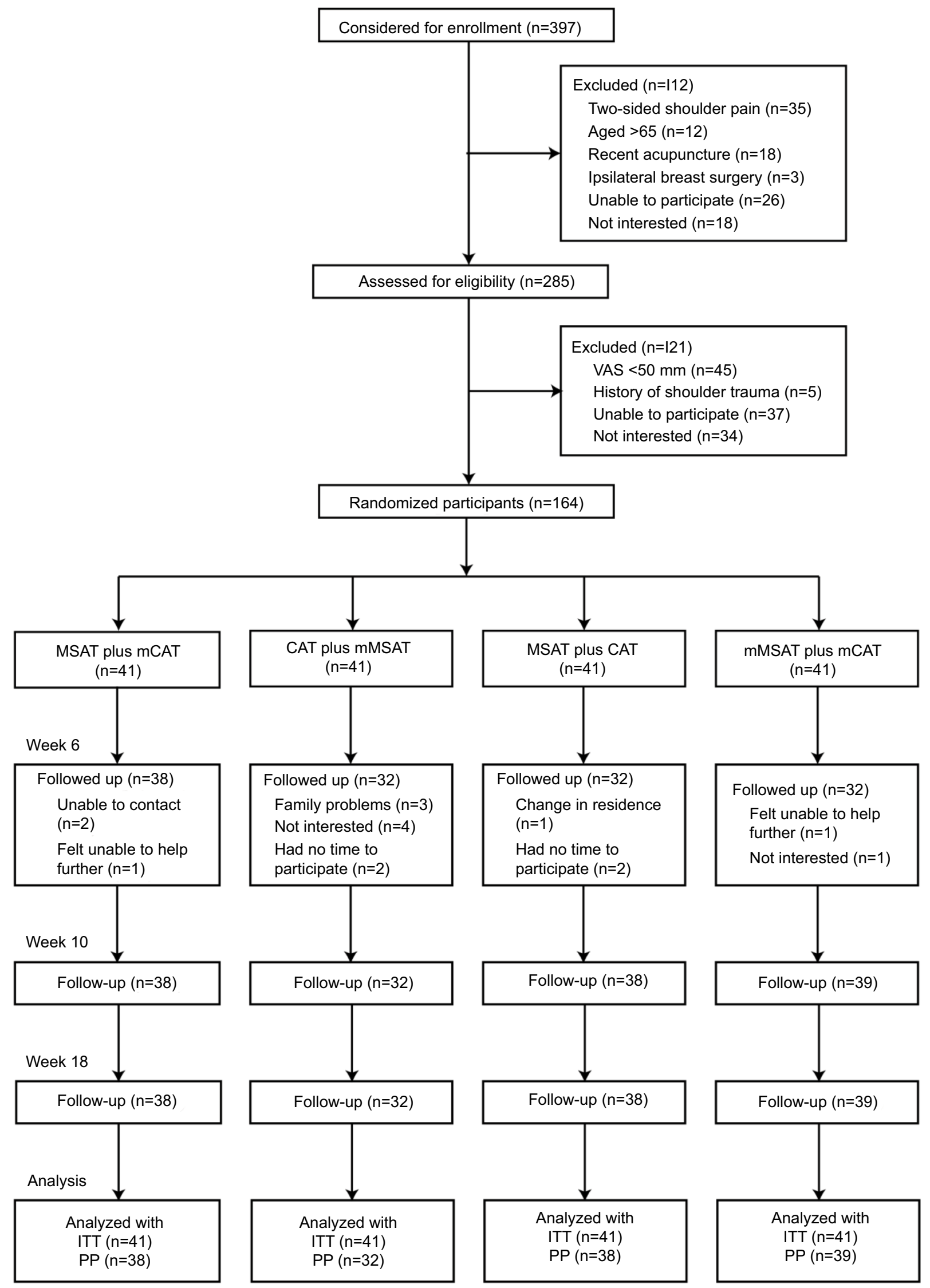

Figure I Flow chart of trial participants.

Abbreviations: CAT, conventional acupuncture therapy; ITT, intention-to-treat; mCAT, minimal CAT; mMSAT, minimal MSAT; MSAT, motion style acupuncture therapy; PP, per protocol; VAS, visual analog scale. 
Table I Baseline characteristics of randomized participants

\begin{tabular}{|c|c|c|c|c|c|}
\hline Characteristics & $\begin{array}{l}\text { MSAT plus } \\
\text { mCAT, } n=41\end{array}$ & $\begin{array}{l}\text { CAT plus } \\
\text { mMSAT, } n=41\end{array}$ & $\begin{array}{l}\text { MSAT plus } \\
\text { CAT, } n=41\end{array}$ & $\begin{array}{l}\text { mMSAT plus } \\
\text { mCAT, } n=4 \text { I }\end{array}$ & $\begin{array}{l}\text { All randomized, } \\
n=164\end{array}$ \\
\hline \multicolumn{6}{|l|}{ Demographic data } \\
\hline Age, years & $51.6(8.9)$ & $54.7(6.6)$ & $55.4(6.9)$ & $54.0(7.8)$ & $53.9(7.7)$ \\
\hline Female & $16(39.0)$ & $30(73.2)$ & $31(75.6)$ & $27(65.9)$ & $104(63.4)$ \\
\hline College graduate & $19(46.3)$ & $17(4 \mid .5)$ & $13(3 \mid .7)$ & $16(39.0)$ & $65(39.6)$ \\
\hline \multicolumn{6}{|l|}{ Previous therapies } \\
\hline Manual & $0(0)$ & $2(4.8)$ & I (2.4) & $3(7.3)$ & $6(3.7)$ \\
\hline Medical & $2(4.8)$ & $6(14.6)$ & $6(14.6)$ & $5(12.2)$ & $19(11.6)$ \\
\hline Duration of illness, years & $0.5(0.5)$ & $0.8(0.6)$ & $0.7(0.5)$ & $0.7(0.5)$ & $0.7(0.6)$ \\
\hline \multicolumn{6}{|l|}{ Affected side } \\
\hline Left & $19(46.3)$ & $18(43.9)$ & $24(58.5)$ & $18(43.9)$ & 79 (48.2) \\
\hline Right & $22(53.7)$ & $23(56.1)$ & $17(4 \mid .5)$ & $23(56.1)$ & $85(51.8)$ \\
\hline Pain intensity VAS & $71.8(12.1)$ & $70.9(14.0)$ & $70.4(13.4)$ & $71.6(12.0)$ & $71.2(1.3)$ \\
\hline Function changes CMS & $50.3(I I .3)$ & $49.6(10.9)$ & $48.9(10.7)$ & $50.2(11.4)$ & $49.7(I I .0)$ \\
\hline \multicolumn{6}{|l|}{ SF-36 } \\
\hline Physical health & $50.4(15.0)$ & $45.0(13.6)$ & $49.8(12.4)$ & $46.6(14.1)$ & $48.0(13.9)$ \\
\hline Mental health & $64.7(19.9)$ & $64.0(20.1)$ & $67.6(21.5)$ & $62.7(21.4)$ & $64.8(20.6)$ \\
\hline
\end{tabular}

Note: All data are presented as number (\%) or mean (SD).

Abbreviations: CAT, conventional acupuncture therapy; CMS, Constant-Murley score; mCAT, minimal CAT; MSAT, motion style acupuncture therapy; mMSAT, minimal MSAT; SF-36, Short Form-36 Health Survey; VAS, visual analog scale.

Table 2 Interaction effects for the outcome measures

\begin{tabular}{|c|c|c|c|c|c|}
\hline Week & $\begin{array}{l}\text { MSAT plus } \\
\text { mCAT, } n=41\end{array}$ & $\begin{array}{l}\text { CAT plus } \\
\text { mMSAT, } n=4 \mid\end{array}$ & $\begin{array}{l}\text { MSAT plus } \\
\text { CAT, } n=41\end{array}$ & $\begin{array}{l}\text { mMSAT plus } \\
\text { mCAT, } n=41\end{array}$ & $P$-value \\
\hline \multicolumn{6}{|c|}{ Pain intensity VAS } \\
\hline 0 & $71.8(12.1)$ & $70.9(14.0)$ & $70.4(13.4)$ & $71.6(12.0)$ & 0.88 \\
\hline 6 & $33.4(19.2)$ & $41.5(24.4)$ & $35.3(25.7)$ & $34.4(24.0)$ & 0.48 \\
\hline 10 & $27.1(21.4)$ & $39.3(22.8)$ & $28.7(24.8)$ & $33.0(33.7)$ & 0.52 \\
\hline 18 & $22.5(23.5)$ & $28.1(24.7)$ & $36.7(25.0)$ & $33.3(24.8)$ & 0.24 \\
\hline \multicolumn{6}{|c|}{ Function changes CMS } \\
\hline 0 & $50.3(11.3)$ & $49.6(10.9)$ & $48.9(10.7)$ & $50.2(11.4)$ & 0.82 \\
\hline 6 & $76.5(12.4)$ & $67.0(15.7)$ & $73.7(17.0)$ & $70.8(15.7)$ & 0.84 \\
\hline 10 & $79.3(11.8)$ & $68.7(16.9)$ & $77.0(18.4)$ & $73.8(15.4)$ & 0.57 \\
\hline 18 & $79.2(14.0)$ & $70.5(16.7)$ & $80.0(17.1)$ & $76.7(16.3)$ & 0.68 \\
\hline \multicolumn{6}{|l|}{ SF-36 } \\
\hline \multicolumn{6}{|c|}{ Physical health } \\
\hline 0 & $50.4(\mid 5.0)$ & $45.0(13.6)$ & $49.8(12.4)$ & $46.6(14.1)$ & 0.83 \\
\hline 6 & $60.0(17.1)$ & $54.7(16.9)$ & $60.8(15.0)$ & $57.2(16.8)$ & 0.52 \\
\hline 10 & $67.7(17.5)$ & $59.0(18.2)$ & $66.8(18.3)$ & $62.6(17.4)$ & 0.62 \\
\hline 18 & $69.1(17.4)$ & $60.7(19.0)$ & $71.2(16.8)$ & $66.1(17.1)$ & 0.17 \\
\hline \multicolumn{6}{|c|}{ Mental health } \\
\hline 0 & 64.7 (19.9) & $64.0(20.1)$ & $67.6(21.5)$ & $62.7(21.4)$ & 0.80 \\
\hline 6 & $70.7(17.5)$ & $66.2(21.6)$ & $72.3(18.2)$ & $67.0(19.0)$ & 0.68 \\
\hline 10 & $75.1(14.7)$ & $69.2(19.0)$ & $74.7(19.6)$ & $69.9(17.7)$ & 0.96 \\
\hline 18 & $74.3(14.8)$ & $68.2(21.9)$ & 78.8 (I4.7) & 74.1 (17.4) & 0.06 \\
\hline
\end{tabular}

Note: All data are presented as mean (SD).

Abbreviations: CAT, conventional acupuncture therapy; mCAT, minimal CAT; MSAT, motion style acupuncture therapy; mMSAT, minimal MSAT; SF-36, Short Form-36 Health Survey; VAS, visual analog scale; CMS, Constant-Murley score.

greater improvement in pain and disability at 10 weeks than those who received mMSAT, and this was maintained over 18 weeks. However, there was no statistically significant difference in the improvement of pain and functional disability between those receiving CAT and those receiving mCAT.
A consensus report characterized a $10 \mathrm{~mm}$ reduction on a $100 \mathrm{~mm}$ VAS as representing a clinically relevant pain improvement. ${ }^{16,17}$ In Molsberger et al's study ${ }^{15} 50 \%$ of reduction compared to baseline in the VAS score evaluating shoulder pain has a clinically significant difference. In 
Table 3 Treatment effectiveness for outcome measures by main treatment effects

\begin{tabular}{|c|c|c|c|c|c|c|}
\hline Outcome measure & MSAT, $n=82$ & mMSAT, $n=82$ & $P$-value & CAT, $n=82$ & mCAT, $n=82$ & $P$-value \\
\hline \multicolumn{7}{|l|}{ Pain intensity VAS } \\
\hline 0 weeks & $71.1(12.8)$ & $71.2(12.9)$ & 0.95 & $70.6(13.6)$ & $71.7(12.0)$ & 0.59 \\
\hline 6 weeks & $34.4(22.5)$ & $37.9(24.4)$ & 0.33 & $38.4(25.1)$ & $33.9(21.5)$ & 0.22 \\
\hline 10 weeks & $27.9(23.0)$ & $36.1(23.4)$ & 0.024 & $34.0(24.3)$ & $30.1(22.6)$ & 0.28 \\
\hline 18 weeks & $25.3(24.1)$ & $35.0(25.0)$ & 0.013 & $29.6(25.2)$ & $30.7(24.7)$ & 0.78 \\
\hline \multicolumn{7}{|l|}{ Function changes CMS } \\
\hline 0 weeks & $49.6(I 1.0)$ & $49.9(11.1)$ & 0.84 & $49.2(10.7)$ & $50.2(11.3)$ & 0.56 \\
\hline 6 weeks & $75.1(14.8)$ & $68.9(15.8)$ & 0.01 & $70.4(16.6)$ & $73.7(14.3)$ & 0.17 \\
\hline 10 weeks & $78.1(15.4)$ & $71.3(16.3)$ & 0.006 & $72.9(18.0)$ & 76.5 (13.9) & 0.14 \\
\hline 18 weeks & 79.9 (I5.5) & $73.3(16.7)$ & 0.010 & $75.3(17.5)$ & $78.0(15.1)$ & 0.29 \\
\hline \multicolumn{7}{|l|}{ Physical health (SF-36) } \\
\hline 0 weeks & $50.1(13.7)$ & $45.8(13.7)$ & 0.05 & $47.4(13.2)$ & $48.5(14.6)$ & 0.61 \\
\hline 6 weeks & $60.4(16.1)$ & $56.0(16.7)$ & 0.09 & $57.8(16.2)$ & $58.6(16.9)$ & 0.74 \\
\hline 10 weeks & $67.2(17.8)$ & $60.8(17.8)$ & 0.023 & $62.9(18.6)$ & $65.1(17.5)$ & 0.43 \\
\hline 18 weeks & $70.1(17.1)$ & $63.4(18.2)$ & 0.015 & $66.0(18.6)$ & $67.6(17.2)$ & 0.55 \\
\hline \multicolumn{7}{|l|}{ Mental health (SF-36) } \\
\hline 0 weeks & $66.1(20.7)$ & $63.4(20.5)$ & 0.39 & $65.8(20.8)$ & $63.7(20.5)$ & 0.51 \\
\hline 6 weeks & 71.5 (I7.9) & $66.6(20.1)$ & 0.10 & $69.3(20.1)$ & $68.9(18.2)$ & 0.89 \\
\hline 10 weeks & $74.9(17.3)$ & $69.6(18.2)$ & 0.06 & $72.0(19.4)$ & $72.5(16.3)$ & 0.85 \\
\hline 18 weeks & $76.6(14.9)$ & $71.2(19.8)$ & 0.05 & 73.5 (19.3) & $74.2(16.0)$ & 0.79 \\
\hline
\end{tabular}

Note: All data are presented as mean (SD).

Abbreviations: CAT, conventional acupuncture therapy; CMS, Constant-Murley score; mCAT, minimal CAT; MSAT, motion style acupuncture therapy; mMSAT, minimal MSAT; SF-36, Short Form-36 Health Survey; VAS, visual analog scale.

Table 4 Patient rating of treatment credibility

\begin{tabular}{lllll}
\hline $\begin{array}{l}\text { Patient's } \\
\text { response }\end{array}$ & $\begin{array}{l}\text { MSAT plus } \\
\text { mCAT, n=38 }\end{array}$ & $\begin{array}{l}\text { CAT plus } \\
\text { mMSAT, } \mathbf{n = 3 2}\end{array}$ & $\begin{array}{l}\text { MSAT plus } \\
\text { CAT, } \mathbf{n = 3 8}\end{array}$ & $\begin{array}{l}\text { mMSAT plus } \\
\text { mCAT, n=39 }\end{array}$ \\
\hline $\begin{array}{l}\text { Confident that treatment can help problem } \\
\text { Very }\end{array}$ & $\mathrm{I}(42.1)$ & $12(37.5)$ & $18(47.4)$ & $17(43.6)$ \\
Quite & $2 \mathrm{I}(55.3)$ & $14(43.8)$ & $19(50.0)$ & $16(41.0)$ \\
Neither & $\mathrm{I}(2.6)$ & $5(15.6)$ & $1(2.6)$ & $5(12.8)$ \\
Not very & $0(0)$ & $\mathrm{I}(3.1)$ & $0(0)$ & $1(2.6)$ \\
Not at all & $0(0)$ & $0(0)$ & $0(0)$ & $0(0)$ \\
\hline
\end{tabular}

Note: All data are presented as number (\%).

Abbreviations: CAT, conventional acupuncture therapy; mCAT, minimal CAT; MSAT, motion style acupuncture therapy; mMSAT, minimal MSAT.

Table 5 Per-protocol analysis for the primary outcome (pain intensity)

\begin{tabular}{lllllll}
\hline Week & MSAT, $\mathbf{n = 7 6}$ & mMSAT, $\mathbf{n = 7 1}$ & $\boldsymbol{P}$-value & CAT, $\mathbf{n = 7 0}$ & mCAT, $\mathbf{n = 7 7}$ & $\boldsymbol{P}$-value \\
\hline 0 & $71.7(12.9)$ & $71.9(13.0)$ & 0.93 & $72.0(13.8)$ & $71.7(12.1)$ & 0.91 \\
6 & $32.1(22.0)$ & $33.5(22.2)$ & 0.71 & $34.2(24.5)$ & $31.4(19.6)$ & 0.45 \\
10 & $25.1(21.6)$ & $31.4(20.7)$ & 0.07 & $29.2(22.3)$ & $27.3(20.3)$ & 0.59 \\
18 & $22.4(22.3)$ & $29.9(22.5)$ & 0.045 & $24.2(22.3)$ & $28.0(22.9)$ & 0.32 \\
\hline
\end{tabular}

Note: All data are presented as mean (SD).

Abbreviations: CAT, conventional acupuncture therapy; mCAT, minimal CAT; MSAT, motion style acupuncture therapy; mMSAT, minimal MSAT.

our study, the primary end points yield similar responder results among the four groups. The results indicate that the improvement in VAS pain intensity with 6-week acupuncture therapy was of a clinically relevant magnitude. In the present study, patients in the four groups who received acupuncture therapy at 6 weeks showed a change in at least 10 points in function improvements after treatment, which was similar to Kukkonen's study. ${ }^{18,19}$

Meta-analyses of 18,000 randomized participants in high-quality trials provided most robust evidence that acupuncture is a reasonable referral option for patients with chronic pain. ${ }^{20}$ Shoulder pain is considered to be one of the 
indications most amenable to treatment with acupuncture. ${ }^{9,14}$ However, a systematic review reported that more research and higher quality research are needed to determine the relative effectiveness of acupuncture approaches in the management of shoulder pain. ${ }^{21}$ Our study has added to this evidence that the novel MSAT, which consists of acupoints far away from the shoulder with a necessary strong Deqi sensation and, most importantly, requires patients to move their shoulder when acupuncture needles are inserted for a certain period of time, is an effective approach for patients with shoulder pain.

Our result is supported by the outcome of Shin et al. They found highly positive effects on pain and function through the collaborative treatment of acupuncture and motion style in acute low back pain patients. ${ }^{9}$ However, unlike the two different acupuncture therapies compared in our study, they compared the motion style acupuncture treatment with a conventional NSAID injection. Our results also concur with a multicenter, randomized controlled trial (425 participants) that reported that acupuncture at acupoints far away from the shoulder in association with physiotherapy improved shoulder function and alleviated pain compared with mock TENS and physiotherapy for patients with a painful shoulder. ${ }^{22}$ Moreover, $\mathrm{Wu}^{23}$ applied acupuncture at the "Houxi" (SI 3), which is an acupuncture point located on the hand, and combined it with lumbar spine movement, producing a strong Deqi sensation and reporting that motion style acupuncture is effective in reducing acute low back pain. However, that study had a low methodological quality with a score of 3 of 10 in the Methodological Quality Assessment by the review authors.

Acupuncture represents a potentially valuable adjunct to the current pain relief strategies, ${ }^{16,24}$ and the knowledge of the mechanism that causes acupuncture-induced analgesia is continuously being accumulated. Endogenous opioid peptides are acknowledged to be involved in peripheral-central mechanisms of acupuncture-induced analgesia. ${ }^{20}$ More recently, studies have focused on the antihyperalgesic effect of acupuncture in inflammatory animal models. ${ }^{25-28}$ With persistent inflammation, neuronal plasticity causes hyperalgesia (hyperexcitable to pain). Several studies demonstrated the presence of local and widespread hyperalgesia in patients with unilateral shoulder pain. ${ }^{29,30}$ Most noteworthy is that a higher degree of widespread sensitization is associated with higher pain perception in subjects with shoulder pain. ${ }^{31}$ This could be one of the potential mechanisms that causing acupoints distal from the shoulder to have efficacy of pain relief.

The main differences between MSAT and mMSAT groups were the location (acupoints vs nonpoints) and the needle manipulation (strong sense of Deqi vs no Deqi).
Neuroimaging evidence has shown that the neurochemical changes within the endogenous m-opioid system and the long-term pain-relieving effects of acupoints were different from those observed during acupuncture at nonpoints, even though they produce almost identical reductions in perceived pain. ${ }^{32,33}$ These studies suggest that acupoints and nonpoints act in different mechanisms. Stronger intensity of Deqi was associated with better therapeutic effects. ${ }^{34}$ The mechanisms underlying the immediate effects on pain reduction and the steady recovery of function in MSAT are still unclear. However, based on previous research, it has been suggested that the strong stimulation of distal acupuncture points in MSAT may enhance the effects of pain relief by triggering "diffuse noxious inhibitory controls" and may increase the secretion of endorphins by stimulating internal activity of the central nervous system. ${ }^{30}$ If patients feel less pain and gain more mobility with encouragement and MSAT treatment, then the treatment could create a positive cycle, leading to heightened therapeutic effects. Nevertheless, the placebo effect can also be considered an active part of the total therapeutic effect. Patients' expectation and placebo responses have been shown to contribute substantially to the effect of acupuncture. ${ }^{36-38}$ Starting a treatment with needles at distant points makes it easy to gain patients' confidence in acupuncture, especially because it can be less painful than needling in the affected region. Although the placebo effect of MSAT may be greater than that of other conventional treatments, if the total therapeutic effects of MSAT are superior, then MSAT could still be considered as a clinically advantageous and valid treatment.

We acknowledge some limitations of the trial. First, the study setting was traditional medicine hospitals and participants visited for the specific purpose of receiving Chinese medicine; therefore, there is a fair possibility that the participants were more favorably inclined toward acupuncture therapy. The participants who received either of the acupuncture interventions in our study were significantly more confident that treatment could help their problem than participants in Foster's study. ${ }^{39}$ Second, because complete blinding was impossible, this study does not allow us to determine whether the observed effectiveness of acupuncture therapy was due to placebo effects, the intensity of provider contact, or the physiologic effect of needling. ${ }^{35}$ Third, acupuncture treatments were not individualized. Our directed acupuncture treatment was uniformly applied. Although not enough evidence has suggested that individualized acupuncture is superior to fixed-point prescriptions, some experts have argued that the latter is suboptimal. ${ }^{30,40}$ Fourth, our findings likely apply to patients with clinically diagnosed shoulder pain but may not 
pertain to selective effects within specific etiology of shoulder pain. Although the Jobe test and drop arm test were chosen as diagnostic tests, a wide spectrum of causes for shoulder pain was enrolled, including myofascial pain and adhesive capsulitis. Future studies should classify etiology for shoulder pain that could make results more convincing. In addition, patients in all groups became better over time and the results might be influenced by the natural course of shoulder pain.

\section{Conclusion}

MSAT had significant and clinically relevant effects when compared with minimal motion style acupuncture or conventional acupuncture in patients with shoulder pain. We now need to assess the long-term effects of MSAT both in comparison to sham interventions and to standard treatment.

\section{Acknowledgments}

The authors involved in this project thank the patients who made this study possible. This research was supported by National Natural Science Foundation for Excellent Young Scholars of China (81222050), Beijing Technology Development of Traditional Chinese Medicine Foundation-Annual Planning Project (JJ 2013-40), and Beijing Municipal Administration of Hospitals Clinical Medicine Development of Special Funding Support (ZYLX201412). The funding source of this study did not have any role in the study design or in conducting the practical work of writing the report.

\section{Disclosure}

The authors report no conflicts of interest in this work.

\section{References}

1. Herin F, Vézina M, Thaon I, Soulat JM, Paris C, ESTEV Group. Predictors of chronic shoulder pain after 5 years in a working population. Pain. 2012;153(11):2253-2259.

2. Whittle S, Buchbinder R. In the clinic. Rotator cuff disease. Ann Intern Med. 2015;162(1):ITC1-ITC15.

3. Sun Y, Chen J, Li H, Jiang J, Chen S. Steroid injection and nonsteroidal anti-inflammatory agents for shoulder pain: a PRISMA systematic review and meta-analysis of randomized controlled trials. Medicine (Baltimore). 2015;94(50):e2216.

4. Papadonikolakis A, McKenna M, Warme W, Martin BI, Matsen FA 3rd. Published evidence relevant to the diagnosis of impingement syndrome of the shoulder. J Bone Joint Surg Am. 2011;93(19):1827-1832.

5. Seida JC, LeBlanc C, Schouten JR, et al. Systematic review: nonoperative and operative treatments for rotator cuff tears. Ann Intern Med. 2010;153(4):246-255.

6. Page MJ, Green S, Kramer S, Johnston RV, McBain B, Buchbinder R. Electrotherapy modalities for adhesive capsulitis (frozen shoulder). Cochrane Database Syst Rev. 2014;(10):CD011324.

7. Green S, Buchbinder R, Hetrick S. Physiotherapy interventions for shoulder pain. Cochrane Database Syst Rev. 2003;(2):CD004258.

8. Page MJ, Green S, Kramer S, et al. Manual therapy and exercise for adhesive capsulitis (frozen shoulder). Cochrane Database Syst Rev. 2014;(8):CD011275.
9. Shin JS, Ha IH, Lee J, et al. Effects of motion style acupuncture treatment in acute low back pain patients with severe disability: a multicenter, randomized, controlled, comparative effectiveness trial. Pain. 2013;154(7):1030-1037.

10. Fu QN, Shi GX, Li QQ, et al. Acupuncture at local and distal points for chronic shoulder pain: study protocol for a randomized controlled trial. Trials. 2014;15:130.

11. Fuchs $\mathrm{S}$, Chylarecki $\mathrm{C}$, Langenbrinck A. Incidence and symptoms of clinically manifest rotator cuff lesions. Int J Sports Med. 1999;20(3):201-205.

12. Jobe FW. Moynes DR delineation of diagnostic criteria and a rehabilitation program for rotator cuff injuries. Am J Sports Med. 1982;10(6):336-339.

13. Linde K, Dincer F. How informed is consent in sham-controlled trials of acupuncture? J Altern Complement Med. 2004;10(2):379-385.

14. Vincent C, Lewith G. Placebo controls for acupuncture studies. J R Soc Med. 1995;88:199-202.

15. Molsberger AF, Schneider T, Gotthardt H, Drabik A. German Randomized Acupuncture Trial for chronic shoulder pain (GRASP)-a pragmatic, controlled, patient-blinded, multi-centre trial in an outpatient care environment. Pain. 2010;151(1):146-154.

16. Madsen MV, Gøtzsche PC, Hróbjartsson A. Acupuncture treatment for pain: systematic review of randomised clinical trials with acupuncture, placebo acupuncture, and no acupuncture groups. BMJ. 2009;338:a3115.

17. Dworkin RH, Turk DC, Wyrwich KW, et al. Interpreting the clinical importance of treatment outcomes in chronic pain clinical trials: IMMPACT recommendations. J Pain. 2008;9(2):105-121.

18. Angst F, Schwyzer HK, Aeschlimann A, Simmen BR, Goldhahn J. Measures of adult shoulder function: disabilities of the Arm, Shoulder, and Hand Questionnaire (DASH) and its short version (QuickDASH), Shoulder Pain and Disability Index (SPADI), American Shoulder and Elbow Surgeons (ASES) Society standardized shoulder assessment form, Constant (Murley) Score (CS), Simple Shoulder Test (SST), Oxford Shoulder Score (OSS), Shoulder Disability Questionnaire (SDQ), and Western Ontario Shoulder Instability Index (WOSI). Arthritis Care Res (Hoboken). 2011;63(Suppl 11):S174-S188.

19. Kukkonen J, Kauko T, Vahlberg T, Joukainen A, Aärimaa V. Investigating minimal clinically important difference for Constant score in patients undergoing rotator cuff surgery. J Shoulder Elbow Surg. 2013;22(12):1650-1655.

20. Vickers AJ, Cronin AM, Maschino AC, et al. Acupuncture for chronic pain: individual patient data meta-analysis. Arch Intern Med. 2012;172:1444-1453.

21. Green S, Buchbinder R, Hetrick S. Acupuncture for shoulder pain. Cochrane Database Syst Rev. 2005;(2):CD005319.

22. Vas J, Ortega C, Olmo V, et al. Single-point acupuncture and physiotherapy for the treatment of painful shoulder: a multicentre randomized controlled trial. Rheumatology (Oxford). 2008;47(6):887-893.

23. Wu Y. Acupuncture for 150 cases of acute lumbago. Shanghai J Acupunct Moxibustion. 1991;10:18-19.

24. Lin JG, Chen WL. Acupuncture analgesia: a review of its mechanisms of actions. Am J Chin Med. 2008;36(4):635-645.

25. Koo ST, Park YI, Lim KS, Chung K, Chung JM. Acupuncture analgesia in a new rat model of ankle sprain pain. Pain. 2002;99(3):423-431.

26. Sekido R, Ishimaru K, Sakita M. Differences of electroacupunctureinduced analgeis effect in normal and inflammatory condition in rats. Am J Chin Med. 2003;31(6):955-965.

27. Huang C, Hu ZP, Long H, Shi YS, Han JS, Wan Y. Attenuation of mechanical but not thermal hyperalgesia by electroacupuncture with the involvement of opioids in rat model of chronic inflammatory pain. Brain Res Bull. 2004;63(2):99-103.

28. Lao L, Zhang RX, Zhang G, Wang X, Berman BM, Ren K. A parametric study of electroacupuncture on persistent hyperalgesia and Fos protein expression in rats. Brain Res. 2004;1020(1-2):18-29.

29. Hidalgo-Lozano A, Fernández-de-las-Peñas C, Alonso-Blanco C, Ge HY, Arendt-Nielsen L, Arroyo-Morales M. Muscle trigger points and pressure pain hyperalgesia in the shoulder muscles in patients with unilateral shoulder impingement: a blinded, controlled study. Exp Brain Res. 2010;202(4):915-925. 
30. Gwilym SE, Oag HC, Tracey I, Carr AJ. Evidence that central sensitisation is present in patients with shoulder impingement syndrome and influences the outcome after surgery. J Bone Joint Surg Br. 2011;93(4):498-502.

31. Coronado RA, Simon CB, Valencia C, George SZ. Experimental pain responses support peripheral and central sensitization in patients with unilateral shoulder pain. Clin J Pain. 2014;30(2):143-151.

32. Harris RE, Zubieta JK, Scott DJ, Napadow V, Gracely RH, Clauw DJ. Traditional Chinese acupuncture and placebo (sham) acupuncture are differentiated by their effects on mu-opioid receptors (MORs). Neuroimage. 2009;47(3):1077-1085.

33. Zubieta JK, Stohler CS. Neurobiological mechanisms of placebo responses. Ann N Y Acad Sci. 2009;1156:198-210.

34. Xu SB, Huang B, Zhang CY, et al. Effectiveness of strengthened stimulation during acupuncture for the treatment of Bell palsy: a randomized controlled trial. CMAJ. 2013;185(6):473-479.
35. White A, Cummings M, Filshie J. An Introduction to Western Medical Acupuncture 7-76. London: Churchill Livingstone Elsevier; 2008.

36. Vase L, Baram S, Takakura N, et al. Specifying the nonspecific components of acupuncture analgesia. Pain. 2013;154(9):1659-1667.

37. White P, Bishop FL, Prescott P, Scott C, Little P, Lewith G. Practice, practitioner, or placebo? A multifactorial, mixed-methods randomized controlled trial of acupuncture. Pain. 2012;153(2):455-462.

38. Bausell B, O'Connell NE. Acupuncture research: placebos by many other names. Arch Intern Med. 2009;169(19):1812-1813.

39. Foster NE, Thomas E, Barlas P, et al. Acupuncture as an adjunct to exercise based physiotherapy for osteoarthritis of the knee: randomised controlled trial. BMJ. 2007;335(7617):436.

40. Paul TM, Soo Hoo J, Chae J, Wilson RD. Central hypersensitivity in patients with subacromial impingement syndrome. Arch Phys Med Rehabil. 2012;93(12):2206-2209. 


\section{Supplementary material}

Table SI Interventions delivered to participants

MSAT: core components

- Acupuncture at "Tiaokou" (ST 38, contralateral), with a strong sense of Deqi

- Allowing an active mobilization of the shoulder

CAT: core components

- Acupuncture at "Jiansanzhen plus Binao" (LI I4) with Deqi

mMSAT: core components

- Acupuncture at nonacupuncture point (contralateral) distal away from the shoulder without Deqi

- Allowing an active mobilization of the shoulder mCAT: core components

- Acupuncture at nonacupuncture points around the shoulder without Deqi

Abbreviations: CAT, conventional acupuncture therapy; mCAT, minimal CAT; mMSAT, minimal MSAT; MSAT, motion style acupuncture therapy.

The Journal of Pain Research is an international, peer reviewed, open access, online journal that welcomes laboratory and clinical findings in the fields of pain research and the prevention and management of pain. Original research, reviews, symposium reports, hypothesis formation and commentaries are all considered for publication.
The manuscript management system is completely online and includes a very quick and fair peer-review system, which is all easy to use. Visit http://www.dovepress.com/testimonials.php to read real quotes from published authors. 